\title{
Bleeding Complications in Ultrasound-Guided Central Venous Access in Patients with Severe Thrombocytopenia: A Propensity Score-Based Analysis
}

Virginia Zarama ( $\sim$ vzarama@hotmail.com )

Fundacion Valle del Lili https://orcid.org/0000-0001-7609-1162

Jorge A. Revelo-Noguera

Fundación Valle del Lili: Fundacion Valle del Lili

Jaime A. Quintero

Fundación Valle del Lili: Fundacion Valle del Lili

Ramiro Manzano

Fundación Valle del Lili: Fundacion Valle del Lili

Francisco L. Uribe-Buriticá

Fundación Valle del Lili: Fundacion Valle del Lili

Daniel F. Carvajal

Fundación Valle del Lili: Fundacion Valle del Lili

Laura M. Ochoa

Fundación Valle del Lili: Fundacion Valle del Lili

Gustavo A. Ospina-Tascón

Fundación Valle del Lili: Fundacion Valle del Lili

Research

Keywords: Thrombocytopenia, central venous catheter, ultrasonography, hemorrhage

Posted Date: August 23rd, 2021

DOl: https://doi.org/10.21203/rs.3.rs-809745/v1

License: (c) (i) This work is licensed under a Creative Commons Attribution 4.0 International License. Read Full License 


\section{Abstract}

Purpose: To study the occurrence of bleeding complications in patients with severe thrombocytopenia (platelet count $<20 \times 10^{3} / \mu \mathrm{L}$ ) subjected to ultrasound-guided central venous access (UG-CVA) while receiving or not routine prophylactic platelet transfusion (PPLT).

Research Question: What is the frequency of bleeding complications related to the placement of ultrasound-guided central venous access in patients with severe thrombocytopenia between 2011 and 2019 at high complexity hospital?

Methods: A total of 221 patients with severe thrombocytopenia subjected to UG-CVA from January 2.011 to November 2.019 were selected. They were classified as positive (P-PPLT) or negative (N-PPLT) recipients of PPLT. Then, P-PPLT $(n=72)$ were 1:1 propensity matched to N-PPLT based on catheter diameter, anatomical insertion site, presence of hematologic malignancy, absolute platelet count and whether the health care provider performing the procedure was an attending or a trainee. Bleeding complications were graded according to the Common Terminology Criteria for Adverse Events (CTCAE) score and adapted to central venous catheter placement. A logistic regression analysis was then performed using "bleeding complications" as a binary compound outcome of major (Grades 3-4) and minor bleeding (Grades 1-2) vs. no bleeding.

Results: Seventy-two patients were classified as P-PPLT, while 149 as N-PPLT. No grades 3-4 of bleeding events were identified in the entire population. No significant differences were observed between N-PPLT and P-PPLT for bleeding Grades 1-2 in both pre-matched (53[35.5\%] vs. 26[36.1\%], p=0.90) and propensity-matched populations $(27[37.5 \%]$ vs. $26[36.1 \%], p=0.80)$. Logistic regression demonstrated that PPLT did not influence any bleeding complication (OR 0.9, 95\% CI 0.42-1.92, p=0.791)

Conclusions: Bleeding complications related to central venous catheterization in acutely ill patients with severe thrombocytopenia are not influenced by routine prophylactic platelet transfusion when catheter placement is performed under ultrasound guidance.

\section{Introduction}

Central venous catheterization is a procedure frequently needed in acutely ill patients. In many cases, acute illness is related with thrombocytopenia and prolonged coagulation times, which increase the risk of bleeding during invasive procedures. In general, rates of bleeding complications associated with insertion of central venous lines range between $1 \%$ and $10 \%$ [1-5]. Nevertheless, the overall risk of major and minor bleeding associated with a central line placement in patients with coagulation abnormalities is highly variable, with bleeding rates ranging between 0 and $32 \%[6-8]$ and no deaths were directly attributed to periprocedural bleeding complications [9-12]. Although some authors claim there is no conclusive evidence about the optimal platelet count before central venous catheter insertion [13], observational studies suggest that counts $>50 \times 10^{3} / \mu \mathrm{L}$ could be safe. Current guidelines recommend prophylactic platelet transfusion (PPLT) before central venous placement in patients with platelet counts 
$<20 \times 10^{3} / \mu \mathrm{L}[6,14]$ or $50 \times 10^{3} / \mu \mathrm{L}[15-16]$. Nevertheless, the strength of such recommendations is weak, and these have been widely based on retrospective observational studies including a very low number of patients with counts $<20 \times 10^{3} / \mu \mathrm{L}[7,17-22]$. Thus, using a predetermined platelet count threshold to routinely use PPLT before central venous catheter placement remains highly debatable and platelet transfusion is not free of risk [23-24].

Ultrasound guidance for central venous catheter placement significantly reduces bleeding complications $[2,5,25-26]$ and its use is recommended by international guidelines [27-29]. Nevertheless, there is not clear information about the safety of ultrasound-guided central venous access (UG-CVA) in severe thrombocytopenic patients without using routine prophylactic platelet transfusion. Thus, we aim to evaluate the occurrence of bleeding complications associated with UG-CVA in acutely ill patients with platelet counts $<20 \times 10^{3} / \mu \mathrm{L}$, receiving or not a prophylactic platelet transfusion.

\section{Methods}

\section{Study population / design}

We retrospectively identified all adult patients $\geq 18$ years old with severe thrombocytopenia (platelet count $<20 \times 10^{3} / \mu \mathrm{L}$ ) of any cause who were subjected to UG-CVA from January 2.011 to November 2.019, in a fourth level university hospital (Fundación Valle del Lili, Cali, Colombia). The local ethical and research committee involving human beings approved this study (Protocol number 1279, IRB/EC approval letter number 210-2018, Fundación Valle del Lili, Cali, Colombia. In agreement with the nature of the study, informed consent was waived.

Clinical and laboratory records were carefully reviewed by the investigators. Patients with platelet counts $>20 \times 10^{3} / \mu \mathrm{L}$ and those in whom central venous cannulation was not ultrasound-guided, were not included for the analysis. Complete demographics, transfusion requirements, catheter size, insertion site, whether the person performing the invasive procedure was an attending or an in-training physician and bleeding complications were collected.

This study was adhered to the standards of the STROBE (Strengthening the Reporting of Observational studies in Epidemiology) guidelines for the report of observational studies [30] and a STROBE statement is provided in Supplementary digital content.

\section{Outcomes}

Primary outcome was the incidence of a combination of major and minor bleeding complications associated with UG-CVA in patients with platelet count $<20 \times 10^{3} / \mu \mathrm{L}$ receiving or not a prophylactic platelet transfusion.

Bleeding complications were graded according to the Common Terminology Criteria for Adverse Events (CTCAE) score and adapted by Zeidler for CVC placement [22, 31-32]. A major bleeding event was 
defined as that requiring any surgical / endovascular / percutaneous intervention, red cell transfusion or associated hemodynamic instability (Grades 3-4). Minor bleeding was defined as oozing from the skin insertion site or hematoma that did not required intervention and resolved with manual compression (Grades 1-2). Additionally, hemoglobin values were analyzed before and 24 hours after the procedure. A decrease in hemoglobin level $\geq 1.5 \mathrm{~g} / \mathrm{dL}$ judged to be associated with the catheter insertion, was also considered a major bleeding event.

\section{Statistical analysis}

Continuous variables were summarized as median and inter-quartile ranges according to their nonnormal distribution. All qualitative variables were expressed as frequencies and percentages. Nonnormally distributed continuous variables were compared by using the Wilcoxon signed-rank test. Categorical variables were compared by using the Pearson $\chi^{2}$ test, the Fisher exact test, or the McNemar test as appropriate. The decision to use a PPLT was always taken by the attending physician according to his clinical judgment. Since patients undergoing UG-CVA were not randomly assigned either to positive prophylactic platelet transfusion (P-PPLT) or negative (N-PPLT), a formal causal inference regarding such platelet transfusion and clinical outcomes of interest is not possible. Thus, we generated a propensity score for each patient using a logistic regression model in order to predict the probability (propensity) of receiving or not PPLT before UG-CVA. Such score was a composite of catheter diameter (13.5Fr vs. 5-8 Fr), venous access site (venous jugular, subclavian or femoral), presence of hematologic malignancy, absolute platelet count, use of anticoagulant and antiaggregant therapy, physician performing the catheter insertion (emergency medicine vs. intensive care specialist and senior vs. in-training physician). After fitting the propensity score, a nearest neighbor-matching algorithm extracted 1:1 matched pairs of P-PPLT and N-PPLT subjects.

A logistic regression analysis was then performed using "bleeding complications" as a binary compound outcome of major (Grades 3-4) and minor bleeding events (Grades 1-2) according with the CTCAE classification for bleeding adapted for central venous catheter placement. In addition, a clearly procedurerelated decrease in hemoglobin levels $>1.5 \mathrm{~g} / \mathrm{dL}$ or an unexplained drop in hemoglobin $>1.5 \mathrm{~g} / \mathrm{dL}$ within the next 24-hours were also considered a major bleeding complication. Logistic regression was conducted including as covariables: site of insertion, type of catheter (gauge), hemato-oncologic disease, $\mathrm{pH}$, previous use of anti-aggregants or anticoagulants, procedural performer training level (in-training vs. faculty); and, platelet count: $<5 \times 10^{3} / \mu \mathrm{L}$, between 5 and $10 \times 10^{3} / \mu \mathrm{L}$, or $>10 \times 10^{3} / \mu \mathrm{L}$. All analyses were performed by using Stata 14.0 (StataCorp. 2015. Stata Statistical Software: Release 14. College Station, TX: StataCorp LP).

\section{Results}

Between January 2011 and November 2019, a total of 16658 patients underwent central venous catheterization. Of these, 16170 patients had a platelet count $\geq 20 \times 10^{3} / \mu \mathrm{L}$ and 167 were not inserted under ultrasound-guidance. Finally, 221 patients were included for the analysis. Selection of patients is 
presented in the Fig. 1. A description of demographics and general characteristics of the overall population is provided in the Table 1. Major bleeding complications (CTCAE grades 3-4) were not observed in the entire cohort, while minor bleeding complications (CTCAE Grades 1-2) were detected in $79(35.7 \%)$ patients. Although $6(2,7 \%)$ patients showed a drop $\geq 1,5 \mathrm{~g} / \mathrm{dL}$ in hemoglobin levels into the 24 hours of catheter placement, a careful review of medical charts revealed that such a decrease was apparently not associated to the catheter insertion itself but mostly due to the own disease course or other bleeding complications. 
Table 1

Clinical Characteristics and outcomes of the entire cohort

\begin{tabular}{|c|c|c|c|c|}
\hline & All $(n=221)$ & $\begin{array}{l}\text { N-PPLT }(n= \\
149)\end{array}$ & $\begin{array}{l}\text { P-PPLT }(n= \\
72)\end{array}$ & $\begin{array}{l}\mathrm{p}- \\
\text { value }\end{array}$ \\
\hline Age, median (IQR) & $49(29-64)$ & $53(32-65)$ & $40(26-56)$ & 0.01 \\
\hline Gender, n (\%) & & & & 0.9 \\
\hline Male & $121(54.7 \%)$ & $82(55 \%)$ & $39(54.1 \%)$ & - \\
\hline Female & $100(45.3 \%)$ & $67(45 \%)$ & $33(45.8 \%)$ & - \\
\hline \multicolumn{5}{|l|}{ Medical History, n (\%) } \\
\hline Heart failure & $6(2.7 \%)$ & $5(3.3 \%)$ & $1(1.4 \%)$ & 0.6 \\
\hline Chronic Liver Disease & $11(5 \%)$ & $7(4.7 \%)$ & $4(5.5 \%)$ & 0.7 \\
\hline Diabetes Mellitus & $21(9.5 \%)$ & $14(9.4 \%)$ & $7(9.7 \%)$ & 0.9 \\
\hline Hypertension & $47(21.2 \%)$ & $36(24.1 \%)$ & $11(15.3 \%)$ & 0.1 \\
\hline Atrial fibrillation & $4(1.8 \%)$ & $3(2 \%)$ & $1(1.4 \%)$ & 1 \\
\hline Chronic Kidney Disease requiring RRT & $6(2.7 \%)$ & $4(2.7 \%)$ & $2(2.8 \%)$ & 1 \\
\hline $\begin{array}{l}\text { Chronic Kidney Disease not requiring } \\
\text { RRT }\end{array}$ & $8(3.6 \%)$ & $7(4.7 \%)$ & $1(1.4 \%)$ & 0.2 \\
\hline Anticoagulation therapy & $5(2.3 \%)$ & $4(2.7 \%)$ & $1(1.4 \%)$ & 1 \\
\hline Antiaggregant therapy & $4(1.8 \%)$ & $4(2.7 \%)$ & $0(0 \%)$ & 0.3 \\
\hline History of Bleeding & $39(17.6 \%)$ & $29(19.5 \%)$ & $10(13.9 \%)$ & 0.3 \\
\hline Acute Kidney Injury at admission & $59(26.7 \%)$ & $42(28.2 \%)$ & $17(23.6 \%)$ & 0.4 \\
\hline Diagnosis at admission $\mathbf{n}(\%)$ & & & & 0.8 \\
\hline Autoimmune disease & $7(3.2 \%)$ & $3(2 \%)$ & $4(5.5 \%)$ & - \\
\hline Hematologic diseases & $37(16.7 \%)$ & $26(17.4 \%)$ & $11(15.3 \%)$ & - \\
\hline Hemato-oncologic diseases & $74(33.5 \%)$ & $48(32.2 \%)$ & $26(36.1 \%)$ & - \\
\hline Hypovolemic shock & $5(2.2 \%)$ & $3(2 \%)$ & $2(2.8 \%)$ & - \\
\hline Hepatic disease & $5(2.2 \%)$ & $3(2 \%)$ & $2(2.8 \%)$ & - \\
\hline Cancer & $9(4 \%)$ & $7(4.7 \%)$ & $2(2.8 \%)$ & - \\
\hline
\end{tabular}

IQR: interquartile range, Cl: confidence interval, RRT: Renal Replacement Therapy, PT: Prothrombin Time, PTT: Partial Thromboplastin Time, INR: International Normalized Ratio, BUN: Blood Urea Nitrogen, CVC: Central Venous Catheter, N-PPLT: Negative Prophylactic Platelet Transfusion, P-PPLT: Positive Prophylactic Platelet Transfusion. 


\begin{tabular}{|c|c|c|c|c|}
\hline & All $(n=221)$ & $\begin{array}{l}\text { N-PPLT }(n= \\
149)\end{array}$ & $\begin{array}{l}\text { P-PPLT }(n= \\
\text { 72) }\end{array}$ & $\begin{array}{l}\mathrm{p}- \\
\text { value }\end{array}$ \\
\hline Renal disease & $3(1.3 \%)$ & $3(2 \%)$ & $0(0 \%)$ & - \\
\hline Acute respiratory distress syndrome & $2(0.9 \%)$ & $2(1.3 \%)$ & $0(0 \%)$ & - \\
\hline Sepsis/ Infectious diseases & $79(37.7 \%)$ & $54(36.2 \%)$ & $25(34.7 \%)$ & - \\
\hline \multicolumn{5}{|l|}{ Laboratory tests, (median) (IQR), n } \\
\hline $\mathrm{pH}$ & $\begin{array}{l}7.36(7.26- \\
7.43), 62\end{array}$ & $\begin{array}{l}7.35(7.26- \\
7.42)\end{array}$ & $\begin{array}{l}7.4(7.26- \\
7.45)\end{array}$ & 0.09 \\
\hline Platelet Count $\left(\times 10^{3} / \mu \mathrm{L}\right)$ & $10(6-14)$ & $11(7-14)$ & $8(5-13)$ & 0.058 \\
\hline PT & $\begin{array}{l}18.1(15-23.3) \\
73\end{array}$ & $\begin{array}{l}18.5(14.5- \\
23.6)\end{array}$ & $\begin{array}{l}17.8(15.7- \\
22.2)\end{array}$ & 0.8 \\
\hline PTT & $\begin{array}{l}33(28.2-45.9) \\
74\end{array}$ & $\begin{array}{l}31.9(28.2- \\
42)\end{array}$ & $\begin{array}{l}36.8(28.8- \\
55.1)\end{array}$ & 0.07 \\
\hline INR & $1.3(1.1-1.8), 73$ & $1.4(1.1-1.8)$ & $1.2(1.2-1.8)$ & 0.5 \\
\hline BUN & $\begin{array}{l}28.7(16-56.7) \\
11\end{array}$ & $\begin{array}{l}30.1(16.1- \\
58.1)\end{array}$ & $\begin{array}{l}28.4(15.7- \\
50.1)\end{array}$ & 0.4 \\
\hline Insertion site, n (\%) & & & & 0.1 \\
\hline Jugular vein & $108(48.9 \%)$ & $66(44.3 \%)$ & $42(58.3 \%)$ & - \\
\hline Subclavian vein & $85(38.4 \%)$ & $63(42.3 \%)$ & $22(30.5 \%)$ & - \\
\hline Femoral vein & $28(12.6 \%)$ & $20(13.4 \%)$ & $8(11.1 \%)$ & - \\
\hline Hospital Ward, n (\%) & & & & 0.1 \\
\hline General hospital ward & $32(14.5 \%)$ & $22(14.7 \%)$ & $10(13.9 \%)$ & - \\
\hline Intensive Care Unit & $104(47 \%)$ & $64(42.9 \%)$ & $40(55.5 \%)$ & - \\
\hline Emergency Department & $85(38.5 \%)$ & $63(42.3 \%)$ & $22(30.6 \%)$ & - \\
\hline Catheter Diameter n (\%) & & & & 0.5 \\
\hline $\begin{array}{l}5-7 \text { Fr (Single, Double and Triple } \\
\text { lumen) }\end{array}$ & $155(70.1 \%)$ & $107(71.8 \%)$ & $48(66.6 \%)$ & - \\
\hline $\begin{array}{l}8 \mathrm{Fr} \text { (Pulmonary Artery Catheter } \\
\text { Introducer) }\end{array}$ & $16(7.2 \%)$ & $9(6 \%)$ & $7(9.7 \%)$ & - \\
\hline
\end{tabular}

IQR: interquartile range, Cl: confidence interval, RRT: Renal Replacement Therapy, PT: Prothrombin Time, PTT: Partial Thromboplastin Time, INR: International Normalized Ratio, BUN: Blood Urea Nitrogen, CVC: Central Venous Catheter, N-PPLT: Negative Prophylactic Platelet Transfusion, P-PPLT: Positive Prophylactic Platelet Transfusion. 


\begin{tabular}{|c|c|c|c|c|}
\hline & All $(n=221)$ & $\begin{array}{l}\text { N-PPLT }(n= \\
149)\end{array}$ & $\begin{array}{l}\text { P-PPLT( }(n= \\
\text { 72) }\end{array}$ & $\begin{array}{l}\mathrm{p}- \\
\text { value }\end{array}$ \\
\hline $\begin{array}{l}\text { 13,5 Fr (Renal Replacement Therapy } \\
\text { Catheter) }\end{array}$ & $50(22.6 \%)$ & $33(22.1 \%)$ & $17(23.6 \%)$ & - \\
\hline \multicolumn{5}{|l|}{ Procedure Provider, n (\%) } \\
\hline Intensive Care & $93(42 \%)$ & $55(36.9 \%)$ & $38(52.8 \%)$ & 0.02 \\
\hline Emergency Medicine & $95(43 \%)$ & $68(45.6 \%)$ & $27(37.5 \%)$ & 0.2 \\
\hline Internal Medicine & $22(9.9 \%)$ & $18(12.1 \%)$ & $4(5.5 \%)$ & 0.1 \\
\hline Other & $11(5 \%)$ & $8(5.3 \%)$ & $3(4.1 \%)$ & 1 \\
\hline $\begin{array}{l}\text { In-traning physicians (Residents or } \\
\text { Fellows) }\end{array}$ & $46(20.8 \%)$ & $25(16.8 \%)$ & $21(29.2 \%)$ & 0.03 \\
\hline $\begin{array}{l}\text { Indication for catheter placement, } \mathbf{n} \\
(\%)\end{array}$ & & & & 0.4 \\
\hline $\begin{array}{l}\text { Infusion of peripheral incompatible } \\
\text { solutions }\end{array}$ & $148(66.9 \%)$ & $102(68.4 \%)$ & $46(63.9 \%)$ & - \\
\hline Inadequate peripheral access & $1(0.4 \%)$ & 0 & $1(1.4 \%)$ & - \\
\hline Renal Replacement Therapy & $43(19.4 \%)$ & $29(19.5 \%)$ & $14(19.4 \%)$ & - \\
\hline Plasmapheresis & $12(5.4 \%)$ & $9(6 \%)$ & $3(4.1 \%)$ & - \\
\hline Cell collection & $2(0.9)$ & $1(0.6 \%)$ & $1(1.4 \%)$ & - \\
\hline Hemodynamic monitoring & $15(6.8 \%)$ & $8(5.3 \%)$ & $7(9.7 \%)$ & - \\
\hline \multicolumn{5}{|l|}{ Complications } \\
\hline Hematomas & $25(11.3 \%)$ & $18(12.1 \%)$ & $7(9.7 \%)$ & 0.6 \\
\hline Hemothorax & 0 & 0 & 0 & - \\
\hline Pneumothorax & 0 & 0 & 0 & - \\
\hline Insertion site bleeding & $69(31.2 \%)$ & $44(29.5 \%)$ & $25(34.7 \%)$ & 0.4 \\
\hline $\begin{array}{l}\text { Drop in hemoglobin after CVC } \\
\text { placement }\end{array}$ & $6(2,7 \%)$ & $5(3.3 \%)$ & $1(1.4 \%)$ & 0.6 \\
\hline \multicolumn{5}{|l|}{ Bleeding complications, n (\%) } \\
\hline Minor bleeding & 79 (35.7\%) & $53(35.5 \%)$ & $26(36.1 \%)$ & 0.9 \\
\hline \multicolumn{5}{|c|}{$\begin{array}{l}\text { IQR: interquartile range, Cl: confidence interval, RRT: Renal Replacement Therapy, PT: Prothrombin } \\
\text { Time, PTT: Partial Thromboplastin Time, INR: International Normalized Ratio, BUN: Blood Urea } \\
\text { Nitrogen, CVC: Central Venous Catheter, N-PPLT: Negative Prophylactic Platelet Transfusion, P-PPLT: } \\
\text { Positive Prophylactic Platelet Transfusion. }\end{array}$} \\
\hline
\end{tabular}


After the propensity-matched procedure, 72 patients were classified as P-PPLT and other 72 as N-PPLT (Table 2). General characteristics of this matched-cohort are showed in the Table 2. Again, no significant differences in minor bleeding complications (CTCAE Grades 1-2) were observed between N-PPLT and PPPLT groups $(27(37.5 \%)$ vs. $26(36.1 \%), p=0.80)$. Logistic regression demonstrated that prophylactic platelet transfusion was not relate $\mathrm{d}$ with prevention of major or minor bleeding complications (OR 0.9, $95 \% \mathrm{Cl} 0.42-1.92, \mathrm{p}=0.791$ ) (Table 3). 
Table 2

Clinical characteristics and outcomes after propensity score matching

\begin{tabular}{|c|c|c|c|c|}
\hline & All $(n=144)$ & $\begin{array}{l}\text { N-PPLT }(n= \\
72)\end{array}$ & $\begin{array}{l}\text { P-PPLT }(n= \\
72)\end{array}$ & $\begin{array}{l}\mathrm{p}- \\
\text { value }\end{array}$ \\
\hline Age, median (IQR) & $40(26-58)$ & $40(24-58.5)$ & $40(26-56)$ & 0.9 \\
\hline \multicolumn{5}{|l|}{ Gender, n (\%) } \\
\hline Male & $78(54.1 \%)$ & $39(54.1 \%)$ & $39(54.1 \%)$ & - \\
\hline Female & $66(45.8 \%)$ & $33(45.8 \%)$ & $33(45.8 \%)$ & - \\
\hline \multicolumn{5}{|l|}{ Medical History, n (\%) } \\
\hline Heart failure & $3(2.1 \%)$ & $2(2.8 \%)$ & $1(1.4 \%)$ & 1 \\
\hline Chronic Liver Disease & $4(2.8 \%)$ & $0(0 \%)$ & $4(5.5 \%)$ & 0.1 \\
\hline Diabetes Mellitus & $12(8.3 \%)$ & $5(6.9 \%)$ & $7(9.7 \%)$ & 0.5 \\
\hline Hypertension & $25(17.3 \%)$ & $14(19.4 \%)$ & $11(15.2 \%)$ & 0.5 \\
\hline Atrial fibrillation & $3(2.1 \%)$ & $2(2.8 \%)$ & $1(1.4 \%)$ & 1 \\
\hline Chronic Kidney Disease requiring RRT & $5(3.4 \%)$ & $3(4.1 \%)$ & $2(2.8 \%)$ & 1 \\
\hline $\begin{array}{l}\text { Chronic Kidney Disease not requiring } \\
\text { RRT }\end{array}$ & $2(1.4 \%)$ & $1(1.4 \%)$ & $1(1.4 \%)$ & 1 \\
\hline Anticoagulation therapy & $2(1.4 \%)$ & $1(1.4 \%)$ & $1(1.4 \%)$ & 1 \\
\hline Antiaggregant therapy & $2(1.4 \%)$ & $2(2.8 \%)$ & $0(0 \%)$ & 0.4 \\
\hline History of Bleeding & $20(13.9 \%)$ & $10(13.9 \%)$ & $10(13.9 \%)$ & 1 \\
\hline Acute Kidney Injury at admission & $33(22.9 \%)$ & $16(22.2 \%)$ & $17(23.6 \%)$ & 0.8 \\
\hline Diagnosis at admission $\mathrm{n}(\%)$ & & & & 0.7 \\
\hline Autoimmune disease & $6(4.1 \%)$ & $2(2.8 \%)$ & $4(5.5 \%)$ & - \\
\hline Hematologic diseases & $26(18.1 \%)$ & $15(20.8 \%)$ & $11(15.3 \%)$ & - \\
\hline Hemato-oncologic diseases & $51(35.4 \%)$ & $25(34.7 \%)$ & $26(33.1 \%)$ & - \\
\hline Hypovolemic shock & $4(2.8 \%)$ & $2(2.8 \%)$ & $2(2.8 \%)$ & - \\
\hline Hepatic disease & $3(2.1 \%)$ & $1(1.4 \%)$ & $2(2.8 \%)$ & - \\
\hline Cancer & $7(4.8 \%)$ & $5(6.9 \%)$ & $2(2.8 \%)$ & - \\
\hline
\end{tabular}

IQR: interquartile range, Cl: confidence interval, RRT: Renal Replacement Therapy, PT: Prothrombin Time, PTT: Partial Thromboplastin Time, INR: International Normalized Ratio, BUN: Blood Urea Nitrogen, CVC: Central Venous Catheter, N-PPLT: Negative Prophylactic Platelet Transfusion, P-PPLT: Positive Prophylactic Platelet Transfusion. 


\begin{tabular}{|c|c|c|c|c|}
\hline & All $(n=144)$ & $\begin{array}{l}\text { N-PPLT }(n= \\
72)\end{array}$ & $\begin{array}{l}\text { P-PPLT }(n= \\
\text { 72) }\end{array}$ & $\begin{array}{l}\mathrm{p}- \\
\text { value }\end{array}$ \\
\hline Renal disease & $1(0.7 \%)$ & $1(1.4 \%)$ & $0(0 \%)$ & - \\
\hline Acute respiratory distress syndrome & $1(0.7 \%)$ & $1(1.4 \%)$ & $0(0 \%)$ & - \\
\hline Sepsis/ Infectious diseases & $45(31.2 \%)$ & $20(27.8 \%)$ & $25(34.7 \%)$ & - \\
\hline \multicolumn{5}{|l|}{ Laboratory tests, (median) (IQR), n } \\
\hline $\mathrm{pH}$ & $\begin{array}{l}7.36(7.26- \\
7.45), 41\end{array}$ & $\begin{array}{l}7.33(7.26- \\
7.42)\end{array}$ & $\begin{array}{l}7.4(7.26- \\
7.45)\end{array}$ & 0.09 \\
\hline 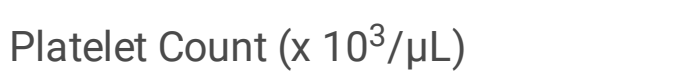 & $9(5.5-13)$ & $10(6-13)$ & $8(5-13)$ & 0.5 \\
\hline PT & $\begin{array}{l}17.9(15.7- \\
23.1), 51\end{array}$ & $\begin{array}{l}18\left(14.6^{-}\right. \\
23.4)\end{array}$ & $\begin{array}{l}17.8(15.7- \\
22.2)\end{array}$ & 0.8 \\
\hline PTT & $\begin{array}{l}32.9(27.5- \\
48.1), 50\end{array}$ & $\begin{array}{l}30.1(27- \\
42.9)\end{array}$ & $\begin{array}{l}36.8(28.8- \\
55.1)\end{array}$ & 0.04 \\
\hline INR & $\begin{array}{l}1.33(1.2-1.8) \\
51\end{array}$ & $\begin{array}{l}1.39(1.17- \\
1.89)\end{array}$ & $\begin{array}{l}1.28(1.2- \\
1.8)\end{array}$ & 0.8 \\
\hline BUN & $\begin{array}{l}28.4(15.7- \\
50.1), 6\end{array}$ & $\begin{array}{l}25(14.2- \\
58.1)\end{array}$ & $\begin{array}{l}28.4(15.7- \\
50.1)\end{array}$ & 0.9 \\
\hline Insertion site, n (\%) & & & & 0.8 \\
\hline Jugular vein & $82(56.9 \%)$ & $40(55.5 \%)$ & $42(58.3 \%)$ & - \\
\hline Subclavian vein & $44(30.5 \%)$ & $22(30.5 \%)$ & $22(30.5 \%)$ & - \\
\hline Femoral vein & $18(12.5 \%)$ & $10(13.9 \%)$ & $8(11.1 \%)$ & - \\
\hline Hospital Ward, n (\%) & & & & 0.8 \\
\hline General hospital ward & $22(15.3 \%)$ & $12(16.6 \%)$ & $10(13.9 \%)$ & - \\
\hline Intensive Care Unit & $78(54.1 \%)$ & $38(52.8 \%)$ & $40(55.5 \%)$ & - \\
\hline Emergency Department & $44(30.5 \%)$ & $22(30.5 \%)$ & $22(30.5 \%)$ & - \\
\hline Catheter Diameter n (\%) & & & & 0.5 \\
\hline $\begin{array}{l}5-7 \mathrm{Fr} \text { (Single, Double and Triple } \\
\text { lumen) }\end{array}$ & $95(66 \%)$ & $47(65.3 \%)$ & $48(66.6 \%)$ & - \\
\hline $\begin{array}{l}8 \mathrm{Fr} \text { (Pulmonary Artery Catheter } \\
\text { Introducer) }\end{array}$ & $11(7.6 \%)$ & $4(5.5 \%)$ & $7(9.7 \%)$ & - \\
\hline
\end{tabular}

IQR: interquartile range, Cl: confidence interval, RRT: Renal Replacement Therapy, PT: Prothrombin Time, PTT: Partial Thromboplastin Time, INR: International Normalized Ratio, BUN: Blood Urea Nitrogen, CVC: Central Venous Catheter, N-PPLT: Negative Prophylactic Platelet Transfusion, P-PPLT: Positive Prophylactic Platelet Transfusion. 


\begin{tabular}{|c|c|c|c|c|}
\hline & All $(n=144)$ & $\begin{array}{l}\text { N-PPLT }(n= \\
72)\end{array}$ & $\begin{array}{l}\text { P-PPLT }(n= \\
72)\end{array}$ & $\begin{array}{l}\mathrm{p} \text { - } \\
\text { value }\end{array}$ \\
\hline $\begin{array}{l}\text { 13,5 Fr (Renal Replacement Therapy } \\
\text { Catheter) }\end{array}$ & $38(26.4 \%)$ & $21(29.1 \%)$ & $17(23.6 \%)$ & - \\
\hline \multicolumn{5}{|l|}{ Procedure Provider, n (\%) } \\
\hline Intensive Care & $77(53.4 \%)$ & $39(54.1 \%)$ & $38(52.8 \%)$ & 0.8 \\
\hline Emergency Medicine & $47(32.6 \%)$ & $20(27.8 \%)$ & $27(37.5 \%)$ & 0.2 \\
\hline Internal Medicine & $12(8.3 \%)$ & $8(11.1 \%)$ & $4(5.5 \%)$ & 0.3 \\
\hline Other & $8(5.5 \%)$ & $5(6.9 \%)$ & $3(4.1 \%)$ & 0.7 \\
\hline $\begin{array}{l}\text { In-traning physicians (Residents or } \\
\text { Fellows) }\end{array}$ & $38(26.4 \%)$ & $17(23.6 \%)$ & $21(29.2 \%)$ & 0.4 \\
\hline $\begin{array}{l}\text { Indication for catheter placement, } \mathbf{n} \\
(\%)\end{array}$ & & & & 0.3 \\
\hline $\begin{array}{l}\text { Infusion of peripheral incompatible } \\
\text { solutions }\end{array}$ & $89(61.8 \%)$ & $43(59.7 \%)$ & $46(63.9 \%)$ & - \\
\hline Inadequate peripheral access & $1(0.7 \%)$ & $0(0 \%)$ & $1(1.4 \%)$ & - \\
\hline Renal Replacement Therapy & $32(22.2 \%)$ & $18(25 \%)$ & $14(19.4 \%)$ & - \\
\hline Plasmapheresis & $10(6.9 \%)$ & $7(9.7 \%)$ & $3(4.2 \%)$ & - \\
\hline Cell collection & $1(0.7 \%)$ & $0(0 \%)$ & $1(1.4 \%)$ & - \\
\hline Hemodynamic monitoring & $11(7.6 \%)$ & $4(5.5 \%)$ & $7(9.7 \%)$ & - \\
\hline \multicolumn{5}{|l|}{ Complications } \\
\hline Hematomas & $13(9 \%)$ & $6(8.3 \%)$ & $7(9.7 \%)$ & 0.7 \\
\hline Hemothorax & 0 & 0 & 0 & * \\
\hline Pneumothorax & 0 & 0 & 0 & * \\
\hline Insertion site bleeding & $50(34.7 \%)$ & $25(34.7 \%)$ & $25(34.7 \%)$ & 1 \\
\hline $\begin{array}{l}\text { Drop in hemoglobin after CVC } \\
\text { placement }\end{array}$ & $2(1.4 \%)$ & $1(1.4 \%)$ & $1(1.4 \%)$ & 1 \\
\hline \multicolumn{5}{|l|}{ Bleeding complications, n (\%) } \\
\hline Minor bleeding & $53(36.8 \%)$ & 27 (37.5\%) & $26(36.1 \%)$ & 0.8 \\
\hline \multicolumn{5}{|c|}{$\begin{array}{l}\text { IQR: interquartile range, Cl: confidence interval, RRT: Renal Replacement Therapy, PT: Prothrombin } \\
\text { Time, PTT: Partial Thromboplastin Time, INR: International Normalized Ratio, BUN: Blood Urea } \\
\text { Nitrogen, CVC: Central Venous Catheter, N-PPLT: Negative Prophylactic Platelet Transfusion, P-PPLT: } \\
\text { Positive Prophylactic Platelet Transfusion. }\end{array}$} \\
\hline
\end{tabular}


Table 3

Logistic Regression for Bleeding Complications

\begin{tabular}{|lllll|}
\hline Variable & Odds Ratio & \multicolumn{2}{l}{$95 \% \mathrm{Cl}$} & P value \\
\hline Platelet Transfusion & 0.90 & 0.42 & 1.92 & 0.791 \\
\hline Jugular Vein Access Site & 1.88 & 0.58 & 6.05 & 0.286 \\
\hline Subclavian Vein Access Site & 0.83 & 0.21 & 3.30 & 0.802 \\
\hline 13,5 Fr catheter & 1.09 & 0.38 & 3.15 & 0.861 \\
\hline Platelet count $<5 \times 10^{3} / \mu \mathrm{L}$ & 0.34 & 0.07 & 1.69 & 0.190 \\
\hline Platelet count $5-10 \times 10^{3} / \mu \mathrm{L}$ & 1.08 & 0.22 & 5.12 & 0.923 \\
\hline Platelet count $10-20 \times 10^{3} / \mu \mathrm{L}$ & 2.63 & 0.42 & 1.64 & 0.300 \\
\hline Hemato-oncologic Disease & 1.59 & 0.77 & 3.29 & 0.205 \\
\hline Anticoagulant Use & 0.40 & 0.03 & 4.53 & 0.464 \\
\hline $\mathrm{pH}$ & 1.24 & 0.65 & 2.34 & 0.093 \\
\hline
\end{tabular}

\section{Discussion}

We can extract two important findings from this observational study: (a) major bleeding complications were not detected in the entire population studied, which reinforces the idea of ultrasound guidance as a safe procedure; (b) prophylactic platelet transfusions were not associated with a reduction of bleeding complications in patients with severe thrombocytopenia subjected to central venous catheterization under ultrasound guidance.

Current guidelines recommend prophylactic platelet transfusion for central venous access in patients with platelet counts $<20 \times 10^{3} / \mu \mathrm{L}[6,14]$ or $50 \times 10^{3} / \mu \mathrm{L}[15-16]$; however, the strength of such recommendations is weak and are largely based on small observational studies including a limited number of patients with very low platelet counts. Previous studies have reported a low rate of bleeding complications in patients with thrombocytopenia undergoing central venous cannulation [7].

Nevertheless, the number of patients with severe thrombocytopenia (platelet counts $<20 \times 10^{3} / \mu \mathrm{L}$ ) included in these studies was extremely low [18-22] and a high proportion of such patients received a prophylactic platelet transfusion prior to the procedure [17, 20-22]. In the largest systematic review of central venous access in patients with severe coagulopathy, Van de Weerdt et al. [7] found no difference in bleeding complications associated with prophylactic platelet transfusions. Remarkably, most patients were subjected to central venous catheterization under ultrasound guidance.

Ultrasound guidance for venous catheter placement has shown to reduce the incidence of bleeding complications [2, 5, 25,33-37] and it is considered a standard of care in clinical practice today [38]. In 
agreement with this, our study included 221 acutely ill patients with severe thrombocytopenia requiring central venous access for any cause (hemodynamic monitoring, medication infusion, renal replacement therapy, etc.), under ultrasound guidance. Remarkably, no major bleeding complications were detected in the entire cohort and the total number of minor bleeding events was quite similar to the previously reported in the literature, which highlights the role of ultrasound as a tool to increase safety in these clinical conditions. These findings were consistent even in the subgroup of patients with platelet counts below $10 \times 10^{3} / \mu \mathrm{L}$. In addition, subclavian access was not associated with an increased rate of bleeding complications compared to the jugular site. Interestingly, procedures without prophylactic platelet transfusion were most commonly performed by emergency medicine physicians, which probably reflects a higher frequency of emergent central venous cannulations in this setting.

This study has several limitations. First, its retrospective nature and, therefore, the lack of control by randomization and blinding might limit the external validity of conclusions. Admittedly, although propensity scores were constructed incorporating baseline characteristics likely influencing the decision for prophylactic platelet transfusions (other than thrombocytopenia itself), other non-identifiable factors might not have been included. In addition, the relatively small sample size of this cohort might introduce a risk of missing important differences at the baseline which might contribute to the absence of difference between the P-PPLT and N-PPLT groups. Second, we were not able to identify if the decision on "non-transfusion" relied on some particular attending physicians, which could constitute a potential factor of confusion. Nevertheless, we speculate that the lower occurrence of prophylactic transfusions ordered by emergency physicians respond to a higher frequency of emergency situations not captured by the retrospective nature of our observation. Third, although ultrasound-guided catheterization is an apparently safe procedure in severe thrombocytopenic patients, appropriate training and developed skills are fundamental to reproduce good results. In our hospital, there is an established ultrasound training program for emergency department and critical care staff, so these findings may not be replicable in other environments.

Fourth, even though our results suggest the apparent safety of non-routine prophylactic transfusion in severe thrombocytopenic patients subjected to venous catheterization under ultrasound guidance, future prospective research efforts are necessary to confirm such results.

\section{Conclusion}

Bleeding complications related to central venous catheterization in acutely ill patients with severe thrombocytopenia are not influenced by routine prophylactic platelet transfusion when catheter placement is performed under ultrasound guidance.

Our findings reinforce the idea about the safety of central venous catheter placement when appropriate ultrasound guidance is used in patients with severe thrombocytopenia and provides more data on the effect of prophylactic platelet transfusion for bleeding complications in this subset of patients. 


\section{Declarations}

Funding: We have not received funding for this study.

Conflicts of interest: The authors have no conflicts of interest to declare.

Ethics approval and consent to participate: The local ethical and research committee involving human beings approved this study (Protocol number 1279, IRB/EC approval letter number 210-2018), Fundación Valle del Lili, Cali, Colombia. In agreement with the nature of the study, informed consent was waived.

Consent for publication: Not applicable.

Availability of data and materials: The data that support the findings of this study are available from the systematic database (BD clinic) of our hospital Fundación Valle del Lili but, restrictions apply to the availability of these data, which were used under license for the current study, and so are not publicly available. Data are however available from the authors upon reasonable request and with permission from Fundación Valle del Lili.

\section{Authors' contributions:}

Concept and design: VZ, GAOT.

Acquisition, analysis or interpretation of data: All authors.

Drafting the manuscript: VZ, JRN, GAOT.

Statistical analysis: RM, GAOT.

Critical revision of the manuscript for important intellectual content and approved the version to be published: All authors.

Acknowledgements: Not applicable.

\section{References}

1. Eisen LA, Narasimhan M, Berger JS, et al. Mechanical complications of central venous catheters. J Intensive Care Med. 2006;21(1):40-6.

2. Karakitsos D, Labropoulos N, De Groot E, et al. Real-time ultrasound-guided catheterization of the internal jugular vein: a prospective comparison with the landmark technique in critical care patients. Crit Care. 2006;10(6):R162.

3. Merrer J, De Jonghe B, Golliot F, et al. Complications of femoral and subclavian venous catheterization in critically ill patients: a randomized controlled trial. JAMA. 2001;286(6):700-7. 
4. Sznajder JI, Zveibil FR, Bitterman H, et al. Central vein catheterization. Failure and complication rates by three percutaneous approaches. Arch Intern Med. 1986;146(2):259-61.

5. Fragou M, Gravvanis A, Dimitriou V, et al. Real-time ultrasound-guided subclavian vein cannulation versus the landmark method in critical care patients: a prospective randomized study. Crit Care Med. 2011;39(7):1607-12.

6. Kaufman RM, Djulbegovic B, Gernsheimer T, et al. Platelet transfusion: a clinical practice guideline from the AABB. Ann Intern Med. 2015;162(3):205-13.

7. Van de Weerdt EK, Biemond BJ, Baake B, et al. Central venous catheter placement in coagulopathic patients: risk factors and incidence of bleeding complications. Transfusion. 2017;57(10):2512-25.

8. Ray Jr CE, Shenoy SS. Patients with thrombocytopenia: outcome of radiologic placement of central venous access devices. Radiology. 1997 Jul;204(1):97-9.

9. Konkle B. Percutaneous interventions in the coagulopathic patient. Semin Intervent Radiol. 2005;22(2):88-94.

10. Tercan F, Ozkan U, Oguzkurt L. US-guided placement of central vein catheters in patients with disorders of hemostasis. Eur $\mathrm{J}$ of Radiol. 2008;65:253-6.

11. Della Vigna P, Monfardini L, Bonomo G, et al. Coagulation disorders in patients with cancer: nontunneled central venous catheter placement with US guidance-a single-institution retrospective analysis. Radiology. 2009;253:249-52.

12. Weigand K, Encke J, Meyer FJ, et al. Low Levels of Prothrombin Time (INR) and Platelets Do Not Increase the Risk of Significant Bleeding when Placing Central Venous Catheters*. Med Klin. 2009;104:331-5.

13. Jodi B, Segal WH, Dzik, Transfusion Medicine/Hemostasis Clinical Trials Network. Paucity of studies to support that abnormal coagulation test results predict bleeding in the setting of invasive procedures: an evidence-based review. Transfusion. 2005;45:1413-25.

14. Schiffer CA, Bohlke K, Delaney M, et al. Platelet Transfusion for Patients With Cancer: American Society of Clinical Oncology Clinical Practice Guideline Update. J Clin Oncol. 2018;36(3):283-99.

15. Patel IJ, Davidson JC, Nikolic B, et al. Consensus guidelines for periprocedural management of coagulation status and hemostasis risk in percutaneous image-guided interventions. J Vasc Interv Radiol. 2012;23(6):727-36.

16. (UK) NCGC. Blood Transfusion. National Institute for Health and Care Excellence (UK). 2015.

17. Barrera R, Mina B, Huang Y, et al. Acute complications of central line placement in profoundly thrombocytopenic cancer patients. Cancer. 1996;78(9):2025-30.

18. DeLoughery TG, Liebler JM, Simonds V, et al. Invasive line placement in critically ill patients: do hemostatic defects matter? Transfusion. 1996;36(9):827-31.

19. Doerfler ME, Kaufman B, Goldenberg AS. Central venous catheter placement in patients with disorders of hemostasis. Chest. 1996;110(1):185-8. 
20. Haas B, Chittams JL, Trerotola So. Large-bore tunneled central venous catheter insertion in patients with coagulopathy. J Vasc Interv Radiol. 2010;21(2):212-7.

21. Tomoyose T, Ohama M, Yamanoha A, et al. Real-time ultrasound-guided central venous catheterization reduces the need for prophylactic platelet transfusion in thrombocytopenic patients with hematological malignancy. Transfus Apher Sci. 2013;49(2):367-9.

22. Zeidler K, Arn K, Senn O, et al. Optimal preprocedural platelet transfusion threshold for central venous catheter insertions in patients with thrombocytopenia. Transfusion. 2011;51(11):2269-76.

23. Vlaar AP, Juffermans NP. Transfusion-related acute lung injury: a clinical review. Lancet. 2013;382(9896):984-94.

24. Kiefel V. Reactions Induced by Platelet Transfusions. Transfus Med Hemother. 2008;35(5):354-8.

25. Wu SY, Ling Q, Cao LH, et al. Real-time two-dimensional ultrasound guidance for central venous cannulation: a meta-analysis. Anesthesiology. 2013;118(2):361-75.

26. Brass $P$, Hellmich $M$, Kolodziej $L$, et al. Ultrasound guidance versus anatomical landmarks for subclavian or femoral vein catheterization. Cochrane Database Syst Rev. 2015;1:CD011447.

27. Apfelbaum JL, Rupp SM, Tung A, et al. Practice guidelines for central venous access: a report by the American Society of Anesthesiologists Task Force on Central Venous Access. Anesthesiology. 2012;116(3):539-73.

28. Lamperti $M$, Bodenham AR, Pittiruti $M$, et al. International evidence-based recommendations on ultrasound-guided vascular access. Intensive Care Med. 2012;38(7):1105-17.

29. Frankel HL, Kirkpatrick AW, Elbarbary M, et al. Guidelines for the Appropriate Use of Bedside General and Cardiac Ultrasonography in the Evaluation of Critically III Patients-Part I: General Ultrasonography. Crit Care Med. 2015;43(11):2479-502.

30. von Elm E, Altman DG, Egger M, Pocock SJ, Gotzsche PC, Vandenbroucke JP, Initiative S. Strengthening the Reporting of Observational Studies in Epidemiology (STROBE) statement: guidelines for reporting observational studies. BMJ. 2007;335(7624):806-8. doi:10.1136/bmj.39335.541782.AD.

31. Basch E, Reeve BB, Mitchell SA, Clauser SB, Minasian LM, et al. Development of the National Cancer Institute's patient-reported outcomes version of the common terminology criteria for adverse events (PRO-CTCAE). J Natl Cancer Inst. 2014 Sep;29(9):dju244. doi:10.1093/jnci/dju244. PMID: 25265940; PMCID: PMC4200059. 106 ) .

32. van Baarle FEHP, van de Weerdt EK, Suurmond B, Müller MCA, Vlaar APJ. Biemond BJ2. Bleeding assessment and bleeding severity in thrombocytopenic patients undergoing invasive procedures. Transfusion. 2020 Mar;60(3):637-49. doi:10.1111/trf.15670. Epub 2020 Jan 31. PMID: 32003910; PMCID: PMC7079124.

33. Leung J, Duffy M, Finckh A. Real-time ultrasonographically-guided internal jugular vein catheterization in the emergency department increases success rates and reduces complications: a randomized, prospective study. Ann Emerg Med. 2006;48(5):540-7. 
34. Hind D, Calvert N, McWilliams R, et al. Ultrasonic locating devices for central venous cannulation: meta-analysis. BMJ. 2003;327(7411):361.

35. Rabindranath KS, Kumar E, Shail R, et al. Ultrasound use for the placement of haemodialysis catheters. Cochrane Database Syst Rev. 2011(11):CD005279.

36. Napolitano M, Malato A, Raffaele F, et al. Ultrasonography-guided central venous catheterisation in haematological patients with severe thrombocytopenia. Blood Transfus. 2013;11(4):506-9.

37. Tercan F, Ozkan U, Oguzkurt L. US-guided placement of central vein catheters in patients with disorders of hemostasis. Eur J Radiol. 2008;65(2):253-6.

38. Shekelle PG, Wachter RM, Pronovost PJ, et al. Making health care safer II: an updated critical analysis of the evidence for patient safety practices. Evid Rep Technol Assess (Full Rep). 2013(211):1-945.

\section{Figures}

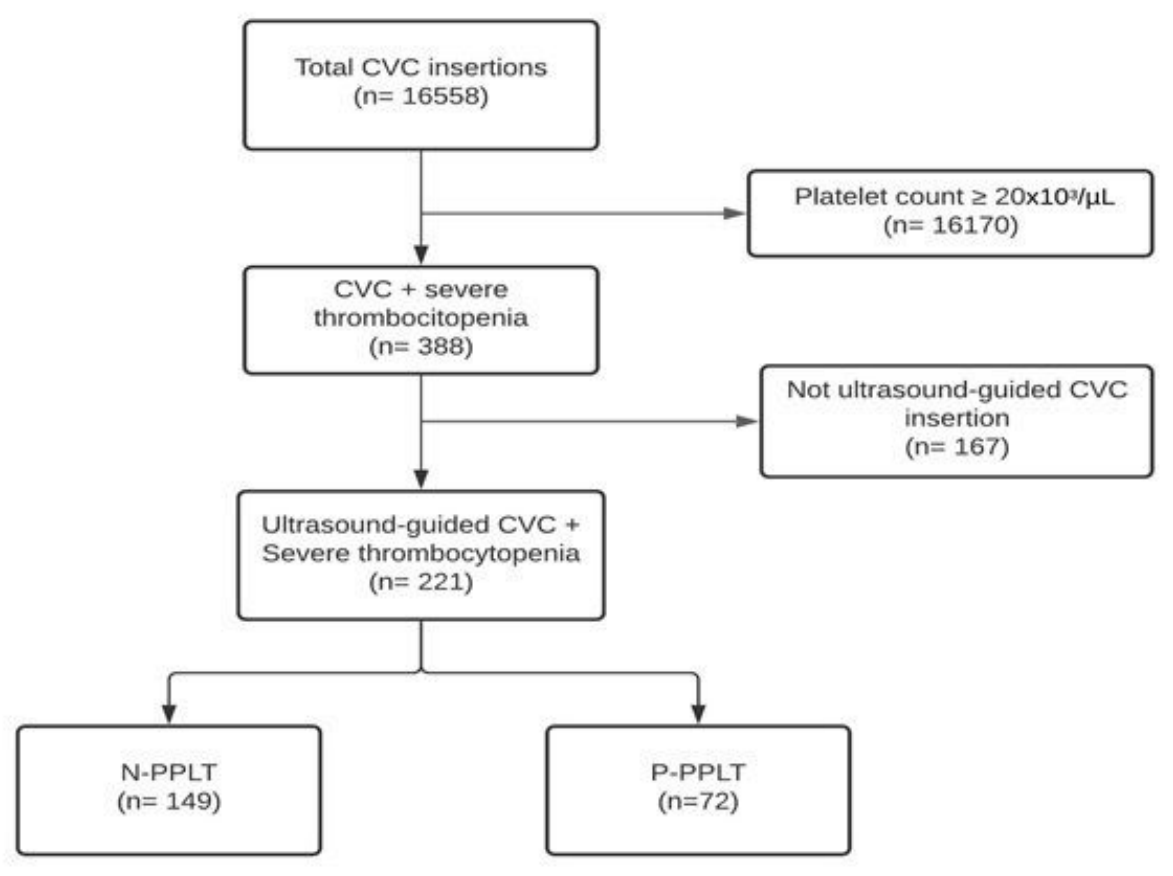




\section{Figure 1}

Study Flowchart. FCVC Central Venous Catheter, N-PPLT Negative Prophylactic Platelet Transfusion, PPPLT Positive Prohylactic Platelet Transfusion

\section{Supplementary Files}

This is a list of supplementary files associated with this preprint. Click to download.

- Suplementarydigitalcontent.STROBEStatement.docx 